\title{
Thermal Neutron Capture Cross Sections of The Palladium Isotopes
}

R. B. Firestone, M. Krticka, D. P. McNabb, B. Sleaford, U. Agvaanluvsan, T. Belgya, Zs. Revay

September 8, 2005

Capture Gamma-Ray Spectroscopy-12 So. Bend, IN, United States September 4, 2005 through September 9, 2005 
This document was prepared as an account of work sponsored by an agency of the United States Government. Neither the United States Government nor the University of California nor any of their employees, makes any warranty, express or implied, or assumes any legal liability or responsibility for the accuracy, completeness, or usefulness of any information, apparatus, product, or process disclosed, or represents that its use would not infringe privately owned rights. Reference herein to any specific commercial product, process, or service by trade name, trademark, manufacturer, or otherwise, does not necessarily constitute or imply its endorsement, recommendation, or favoring by the United States Government or the University of California. The views and opinions of authors expressed herein do not necessarily state or reflect those of the United States Government or the University of California, and shall not be used for advertising or product endorsement purposes. 


\title{
THERMAL NEUTRON CAPTURE CROSS SECTIONS OF THE PALLADIUM ISOTOPES
}

\author{
R.B. Firestone*, M. Krtička ${ }^{\dagger}$, D.P. McNabb ${ }^{* *}$, B. Sleaford ${ }^{* *}$, U. Agvaanluvsan** \\ and T. Belgya ${ }^{\ddagger}$ and Zs. Revay \\ *Lawrence Berkeley National Laboratory, Berkeley, CA 94720 \\ ${ }^{\dagger}$ Charles University in Prague, Faculty of Mathematics and Physics, V Holešovickách 2, CZ-180 00 Prague 8 , \\ Czech Republic \\ ** Lawrence Livermore National Laboratory, Livermore, California 94551 \\ ${ }^{\ddagger}$ Institute of Isotope and Surface Chemistry, H-1525, Budapest, Hungary
}

\begin{abstract}
We have measured precise thermal neutron capture $\gamma$-ray cross sections $\sigma_{\gamma}$ for all stable Palladium isotopes with the guided thermal neutron beam from the Budapest Reactor. The data were compared with other data from the literature and have been evaluated into the Evaluated Gamma-ray Activation File (EGAF)[1]. Total radiative neutron capture cross-sections $\sigma_{0}$ can be deduced from the sum of transition cross sections feeding the ground state of each isotope if the decay scheme is complete. The Palladium isotope decay schemes are incomplete, although transitions deexciting low-lying levels are known for each isotope. We have performed Monte Carlo simulations of the Palladium thermal neutron capture de-excitation schemes using the computer code DICEBOX [2]. This program generates a level scheme where levels below a critical energy $E_{c r i t}$ are taken from experiment, and those above $E_{c r i t}$ are calculated by a random discretization of an a priori known level density formula $\rho\left(E, J^{\pi}\right)$. Level de-excitation branching intensities are taken from experiment for levels below $E_{c r i t}$ and the capture state, or calculated for levels above $E_{c r i t}$ assuming an a priori photon strength function and applying allowed selection rules and a Porter-Thomas distribution of widths. The calculated feeding to levels below $E_{\text {crit }}$ can then be normalized to the measured cross section deexciting those levels to determine the total radiative neutron cross-section $\sigma_{0}$. In this paper we have measured $\sigma_{0}\left[{ }^{102} \operatorname{Pd}(\mathrm{n}, \gamma)\right]=0.9 \pm 0.3 \mathrm{~b}, \sigma_{0}\left[{ }^{104} \operatorname{Pd}(\mathrm{n}, \gamma)\right]=0.61 \pm 0.11 \mathrm{~b}$, $\sigma_{0}\left[{ }^{105} \operatorname{Pd}(\mathrm{n}, \gamma)\right]=21.1 \pm 1.5 \mathrm{~b}, \sigma_{0}\left[{ }^{106} \mathrm{Pd}(\mathrm{n}, \gamma)\right]=0.36 \pm 0.05 \mathrm{~b}, \sigma_{0}\left[{ }^{108} \mathrm{Pd}(\mathrm{n}, \gamma)(0)\right]=7.6 \pm 0.6 \mathrm{~b}, \sigma_{0}\left[{ }^{108} \operatorname{Pd}(\mathrm{n}, \gamma)(189)\right]=0.185 \pm 0.011$ $\mathrm{b}$, and $\sigma_{0}\left[{ }^{110} \mathrm{Pd}(\mathrm{n}, \gamma)\right]=0.10 \pm 0.03 \mathrm{~b}$. We have also determined from our statistical calculations that the neutron capture state in ${ }^{107} \mathrm{Pd}$ is best described as $2^{+}(60 \%)+3^{+}(40 \%)$. Agreement with literature values was excellent in most cases. We found significant discrepancies between our results for ${ }^{102} \mathrm{Pd}$ and ${ }^{110} \mathrm{Pd}$ and earlier values that could be resolved by re-evaluation of the earlier results.
\end{abstract}

\section{INTRODUCTION}

Total radiative thermal neutron capture cross sections are usually determined by measuring either the neutron transmission rate through a target or the activation rate of radioactive product. Both methods require either knowledge of the neutron flux or use of a comparator material of well-known cross section. Transmission rates can be subject to significant corrections for neutron scattering and geometrical uncertainties in the positioning of target and comparator materials. Activation measurements are usually more accurate, but they also require normalization to a comparator, typically gold, and they rely on the accuracy of the decay scheme normalization.

In this paper we report a new method for the determination total radiative thermal neutron cross sections using prompt neutron capture $\gamma$-rays measured with guided neutron beams at the Budapest Reactor. Prompt $\gamma$-rays offer the advantage that the cross section is determined by both the total primary transition cross section deexciting the capture state and the total secondary transition cross section feeding the ground state. Complete decay schemes can be determined for very light isotopes, and complete measurement of secondary $\gamma$-ray cross sections feeding the ground state can be measured for most isotopes of elements up to Fe. For heavier or low abundance isotopes, the measurements are generally incomplete.

For the more complex decay schemes of heavier elements, the contribution from unobserved continuum $\gamma$ rays feeding the ground state must be determined to obtain the total radiative cross section. In this paper we have used the Monte Carlo computer code DICEBOX [2] to calculate "complete" neutron capture decay schemes for the Palladium isotopes. These decay schemes are then normalized using measured cross sections deexciting low-lying levels in the Palladium isotopes to deter- 
mine the total radiative cross sections.

\section{EXPERIMENTAL}

Neutron capture $\gamma$-ray cross sections for elemental targets with $Z=1-84,90,92$ have been measured at the Budapest Reactor with the $2 \times 10^{6} \mathrm{n} / \mathrm{s}$ guided thermal neutron beam[3]. The target station is located far from the reactor where both primary and secondary $\gamma$-rays can be measured in low background conditions. Cross sections were measured using either stoichiometric compounds or accurately prepared mixtures containing the standard elements $\mathrm{H}, \mathrm{N}$, or $\mathrm{Cl}$ whose $\gamma$-ray cross sections are precisely known. The $\gamma$-ray cross sections were then accurately determined from their intensity relative to the standard $\gamma$-ray transitions of the comparators.

An elemental target consisting of $1.6 \mathrm{~g}$ of $\mathrm{PdCl}_{2}$ with a thickness of $0.4 \mathrm{~g} / \mathrm{cm}^{2}$ was irradiated in the $2 \times 10^{6}$ $\mathrm{n} \cdot \mathrm{s}^{-1} \mathrm{~cm}^{-2}$ guided thermal neutron beam at Budapest Reactor for 8753 seconds. Prompt gamma-rays from the target were detected with a $25 \%$ efficient, Comptonsuppressed, HPGe detector. Counting efficiency was determined over the range of $50 \mathrm{keV}$ to $10 \mathrm{MeV}$ with radioactive sources and $(\mathrm{n}, \gamma)$ reaction gamma rays to a precision of better that $1 \%$ from $500 \mathrm{keV}$ to $6 \mathrm{MeV}$ and better than $3 \%$ at all energies[4]. The $\gamma$-ray spectra were analyzed using the Hypermet PC program [5]. A total of $202 \gamma$-rays were assigned to were assigned to the six Palladium isotopes ${ }^{103} \mathrm{Pd},{ }^{105} \mathrm{Pd},{ }^{106} \mathrm{Pd},{ }^{107} \mathrm{Pd},{ }^{109} \mathrm{Pd}$, and ${ }^{111} \mathrm{Pd}$ on the basis of energy and intensity by comparison with data from the ENSDF [6] file. The $\gamma$-ray cross section data were sufficient to determine the deexcitation cross sections $\sigma$ (total) for 101 Palladium levels including at least one level from each isotope. These results are summarized in Table 1.

\section{DICEBOX CALCULATIONS}

Theoretical statistical feedings to low-lying levels have been calculated using the computer code DICEBOX [2]. This code determines the theoretical uncertainty in the level feedings due to statistical fluctuations using an algorithm based on the extreme statistical model of nucleus. Below a critical energy $E_{\text {crit }}$ the level scheme, i.e. energies, spins and parities of all levels and all depopulating transitions, is assumed to be known from experiment. Above this energy, an unknown set of levels is determined by a random discretization of a level density formula $\rho\left(E, J^{\pi}\right)$. The partial radiative width $\Gamma_{a \gamma b}$ for $\gamma$ ray decay with an energy $E_{\gamma}$ from a level $a$ to a level $b$ is assumed to vary with a Porter-Thomas distribution whose mean value is determined by the level density and a photon strength function $S\left(E_{\gamma}\right)$ given by

$$
\Gamma_{a \gamma b}=\frac{S\left(E_{\gamma}\right) \times E_{\gamma}^{3}}{\rho\left(E_{a}, J_{a}^{\pi}\right)},
$$

where $\rho\left(\mathrm{E}_{a}, \mathrm{~J}_{a}^{\pi}\right)$ is the level density at initial level $a$. Two choices of level density models are available with DICEBOX. The constant temperature model (CTF) [7] is given by

$$
\rho\left(E_{a}, J_{a}^{\pi}\right)=f_{J} \frac{1}{T} e^{\left(E-E_{0}\right) / T}
$$

where $T$ is the nuclear temperature and $E_{0}$ is the backshift. The back-shifted Fermi gas model (BSFG) $[7,8,9]$ is given by

$$
\rho\left(E_{a}, J_{a}^{\pi}\right)=\frac{f_{J} e^{2 \sqrt{a\left(E-E_{1}\right)}}}{12 \sqrt{2} \sigma a^{\frac{1}{4}}\left(E-E_{1}\right)^{\frac{5}{4}}}
$$

where $a$ is the conventional shell-model level-density parameter and $E_{1}$ is another back shift. The factor $f_{J}$ represents the probability that a randomly chosen level has spin $J[7]$ and is given by

$$
f_{J}=\frac{2 J+1}{2 \sigma^{2}} e^{-\left(J+\frac{1}{2}\right)^{2} / 2 \sigma^{2}}
$$

where $\sigma=0.98 A^{0.29}$ for the CTF model and $\sigma=$ $0.298 A^{1 / 3} a^{1 / 4}\left(E-E_{1}\right)^{1 / 4}$ for the BSFG model. The parameters for both the CTF and BSFG models are determined from the known level below $E_{c r i t}$ and resonance data for levels above the neutron capture state. Neither the CTF or BSFG model was found to give significantly different results in these calculations.

The photon strength function $S^{X L}\left(E_{\gamma}\right)$ for multipolarities XL is assumed to be independent of spin and parity and is defined as

$$
S^{X L}\left(E_{\gamma}\right)=\frac{1}{E_{\gamma}^{2 L+1}} \frac{\left\langle\Gamma_{a b}^{(J)}\right\rangle_{a}}{\left\langle D_{J}\right\rangle}
$$

Here $\left\langle\Gamma_{a b}^{(J)}\right\rangle_{a}$ represents the value of the partial radiation width $\Gamma_{a b}^{(J)}$ for a transition $a \rightarrow b$, averaged over initial levels $a$ with a fixed parity $\pi$ and spin $J . D_{J}$ is the average spacing between initial levels. Only the lowest-order multipolarities E1, M1, and E2 are included in these calculations. Numerous options for calculating $S^{X L}\left(E_{\gamma}\right)$ including single-particle, Axel and Brink [10], Kopecky and Chrien [11], Kadmenskij et al [12], and various semi-empirical models are available in DICEBOX. The choice of model did not significantly affect our results.

DICEBOX constructs a complete, artificial decay scheme and then randomly simulates $\gamma$-ray cascades. Selection rules for different types of transitions are taken 
TABLE 1. Experimental neutron capture cross sections feeding excited states in Palladium isotopes. Ground state feedings for ${ }^{106,109} \mathrm{Pd}$ were determined from experimental levels scheme for all levels below $E_{\text {crit }}$.

\begin{tabular}{|c|c|c|c|c|c|c|c|}
\hline Isotope & $\begin{array}{c}\text { E(Level) } \\
(\mathrm{keV})\end{array}$ & $\mathbf{J}^{\pi}$ & $\begin{array}{c}\sigma(\text { total }) \\
\text { (barns) }\end{array}$ & $\begin{array}{c}\mathrm{E}_{\text {crit }} \\
(\mathrm{keV})\end{array}$ & $\begin{array}{l}\text { Feeding } \\
(\%)\end{array}$ & $\begin{array}{c}\sigma_{\gamma}\left(\begin{array}{c}\text { This work }) \\
\text { (barns) }\end{array}\right. \\
\end{array}$ & $\begin{array}{c}\sigma_{\gamma}(\text { Literature })^{*} \\
(\text { barns })\end{array}$ \\
\hline${ }^{103} \mathrm{Pd}$ & $118.736(17)$ & $3 / 2+$ & $0.51(14)$ & 500 & $58(8)$ & $0.9(3)$ & $1.82(20)^{\dagger}$ \\
\hline${ }^{105} \mathrm{Pd}$ & $\begin{array}{c}280.51(22) \\
306.25(3) \\
344.512(18) \\
560.75(3) \\
644.53(4) \\
\text { Average }\end{array}$ & $\begin{array}{l}3 / 2+ \\
7 / 2+ \\
1 / 2+ \\
3 / 2+ \\
7 / 2-\end{array}$ & $\begin{array}{c}0.145(13) \\
0.040(8) \\
0.099(18) \\
0.050(10) \\
0.063(6)\end{array}$ & 680 & $\begin{array}{c}30(8) \\
3.9(14) \\
15(5) \\
6.5(15) \\
3.8(20)\end{array}$ & $\begin{array}{c}0.48(14) \\
1.02(42) \\
0.66(31) \\
0.77(24) \\
1.7(9) \\
0.61(11)\end{array}$ & $0.6(3)$ \\
\hline${ }^{106} \mathrm{Pd}$ & 0 & $0+$ & $20.0(3)$ & 2505 & $94.8(15)$ & 21.1(15) & $21.0(15)$ \\
\hline${ }^{107} \mathrm{Pd}$ & $\begin{array}{c}115.74(12) \\
302.78(15) \\
312.20(10) \\
381.80(13) \\
471.21(24) \\
\text { Average }\end{array}$ & $\begin{array}{c}1 / 2+ \\
5 / 2+ \\
7 / 2+ \\
3 / 2+ \\
(3 / 2)+\end{array}$ & $\begin{array}{l}0.095(9) \\
0.046(4) \\
0.024(4) \\
0.043(6) \\
0.024(5)\end{array}$ & 480 & $\begin{array}{c}28(7) \\
8.5(30) \\
3.2(22) \\
11(2) \\
8(2)\end{array}$ & $\begin{array}{c}0.34(9) \\
0.54(20) \\
0.8(5) \\
0.39(9) \\
0.30(10) \\
0.36(5)\end{array}$ & $0.29(3)$ \\
\hline${ }^{109} \mathrm{Pd}$ & $\begin{array}{c}0 \\
188.990(10)\end{array}$ & $\begin{array}{l}5 / 2+ \\
11 / 2-\end{array}$ & $\begin{array}{c}5.93(8) \\
0.185(11)\end{array}$ & 350 & $\begin{array}{c}78(6) \\
100\end{array}$ & $\begin{array}{c}7.6(6) \\
0.185(11)\end{array}$ & $\begin{array}{c}7.6(4) \\
0.18(3)\end{array}$ \\
\hline${ }^{111} \mathrm{Pd}$ & $\begin{array}{l}\text { 191.3(3) } \\
\text { 195.1(2) } \\
\text { Average }\end{array}$ & $\begin{array}{c}{[3 / 2]+} \\
[3 / 2)]+\end{array}$ & $\begin{array}{l}0.016(4) \\
0.019(8)\end{array}$ & 200 & $\begin{array}{l}18(5) \\
18(4)\end{array}$ & $\begin{array}{l}0.09(3) \\
0.11(5) \\
0.10(3)\end{array}$ & $0.19(3)$ \\
\hline
\end{tabular}

* from reference [13] except where noted

$\dagger$ from reference [14]

into account. One unique choice of a level scheme and all partial radiative widths is termed a nuclear realization. As there are an infinite number of possible nuclear realizations, the simulated population of low-lying levels is subject to fluctuations. If the intensities of primary transitions to low-lying levels are known, as in the case of ${ }^{106} \mathrm{Pd}$ and ${ }^{109} \mathrm{Pd}$, they are taken from experiment. Otherwise they are simulated and their values will differ from realization to realization.

The calculated deexcitation branchings feeding the observed levels in the Palladium isotopes for the indicated values of $\mathrm{E}_{\text {crit }}$ is given in Table 1. The uncertainties in these values were determined from the variations in the multiple realizations of each decay scheme. Extensive data for many levels in ${ }^{106} \mathrm{Pd}$ and ${ }^{109} \mathrm{Pd}$ were recorded, and for these cases the calculated branching percentage for the sum of secondary transition cross sections feeding the ground state is reported in Table 1 . The total radiative neutron capture cross sections $\sigma_{\gamma}$ in Table 1 are given by the ratio $100 \times \sigma($ total $) / \%$ Feeding.

\section{RESULTS}

The total radiative neutron capture cross sections for the Palladium isotopes derived from the experimental cross sections feeding the levels and the relative branching intensities calculated with DICEBOX feeding these levels is shown in Table 1 . For ${ }^{102} \operatorname{Pd}(\mathrm{n}, \gamma)$ we measured $\sigma_{\gamma}=0.9 \pm 0.3 \mathrm{~b}$ based on the de-excitation cross section of the first excited state in ${ }^{103} \mathrm{Pd}$. This value is considerably lower than $\sigma_{\gamma}=1.82 \pm 0.20 \mathrm{~b}$ from Duncan and Krane [14]. That value was measured assuming $\mathrm{P}_{357.4 \gamma}=0.0221 \pm 0.0007 \%$ for ${ }^{103} \mathrm{Pd}$ decay from reference [15]. A previous measurement by Zoller et al [16] gave $\mathrm{P}_{357.4 \gamma}=0.0324 \pm 0.0016 \%$ leading to $\sigma_{\gamma}=1.24 \pm 0.15$ $\mathrm{b}$ which is in better agreement with our value.

For ${ }^{104} \mathrm{Pd}(\mathrm{n}, \gamma)$ we determined that $\sigma_{\gamma}=0.61 \pm 0.11 \mathrm{~b}$ based on the average population of five levels in ${ }^{105} \mathrm{Pd}$. This value is in good agreement with $\sigma_{\gamma}=0.6 \pm 0.3 \mathrm{~b}$ from Mughabghab et al [13]. Similarly, For ${ }^{106} \mathrm{Pd}(\mathrm{n}, \gamma)$ we determined that $\sigma_{\gamma}=0.36 \pm 0.05 \mathrm{~b}$ from the average population of five levels in ${ }^{107} \mathrm{Pd}$ in agreement with $\sigma_{\gamma}=0.29 \pm 0.03 \mathrm{~b}$ from Ref. 13. The experimental value $\sigma_{\gamma}=0.10 \pm 0.03 \mathrm{~b}$ for ${ }^{110} \mathrm{Pd}(\mathrm{n}, \gamma)$, based on the observance 
of two levels in ${ }^{111} \mathrm{Pd}$, is lower than the compiled value of $\sigma_{\gamma}=0.19 \pm 0.03 \mathrm{~b}$ which is from Sehgal et al [17]. Comparison of other cross sections measured in Ref. 17 indicates that they were typically about twice as large as the values compiled in Ref. 13. Presumably this discrepancy resulted from an imprecise determination of the neutron flux in Ref. 17. The Sehgal et al value can be renormalized by a factor of 0.54 , necessary to correct their measurement of $14 \pm 2 b$ for the ${ }^{108} \operatorname{Pd}(n, \gamma)$ cross section, yielding $\sigma_{\gamma}=0.10 \pm 0.02 \mathrm{~b}$, which agrees with our result.

Our values of $\sigma_{\gamma}=21.1 \pm 1.5 \mathrm{~b}$ for ${ }^{105} \mathrm{Pd}(\mathrm{n}, \gamma)$ and $\sigma_{\gamma}=7.6 \pm 0.6 \mathrm{~b}$ for ${ }^{108} \mathrm{Pd}(\mathrm{n}, \gamma)$ were determined using data for many levels and are in excellent agreement with the compiled values from Ref. 13. In addition, a new value has been determined for the cross section feeding the 4.7 min isomer in ${ }^{109} \mathrm{Pd} \sigma_{\gamma}\left[{ }^{108} \mathrm{Pd}(\mathrm{n}, \gamma)(189)\right]=0.185 \pm 0.011$ $\mathrm{b}$, determined by measuring the $188.99-\mathrm{keV} \gamma$-ray cross section in equilibrium with the prompt transitions. Calculation of ${ }^{106} \mathrm{Pd}$ statistical feeding was complicated by the unknown relative contributions of capture states with spins $2^{+}$, and $3^{+}$. Figures 1 and 2 show comparisons of the experimental and statistical side feedings for each $\mathrm{J}^{\pi}$ assignment. Agreement between experiment and theory in both cases is poor in both cases. We then solved for the mixing fraction of capture state spins that minimizes the $\chi^{2} / f$ comparison between experiment and theory and determined that $2^{+}(60 \%)+3^{+}(40 \%)$ gave an excellent fit, as is shown in Figure 3. The good agreement between experimental and statistical side feedings for ${ }^{109} \mathrm{Pd}$, where the capture state is uniquely $1 / 2^{+}$, is shown in Figure 4.

New measurements of the total thermal neutron radiative cross sections for ${ }^{102,104,105,106,108,110} \operatorname{Pd}(n, \gamma)$ have been performed using prompt $\gamma$-ray cross sections to renormalize statistical model calculations using DICEBOX. These results had comparable precision to previous values. Our measurements provide independent confirmation of previous results and can be used to identify and resolve problems with earlier experiments. A large library of prompt neutron capture $\gamma$-ray data has been measured for all other stable elements and will be analyzed in the future to determine additional total radiative cross sections.

\section{ACKNOWLEDGEMENTS}

This work was supported by grants from the Director, Office of Science, Office of Basic Energy Sciences, of the U.S. Department of Energy under Contract No. DEAC03- 76SF00098; the U.S. Department of Energy by University of California, Lawrence Livermore National Laboratory under Contract W-7405-Eng-48; NNSA Academic Alliance grant No. DE-FG03-03NA00076, and the Grant Agency of the Czech Republic under Contract

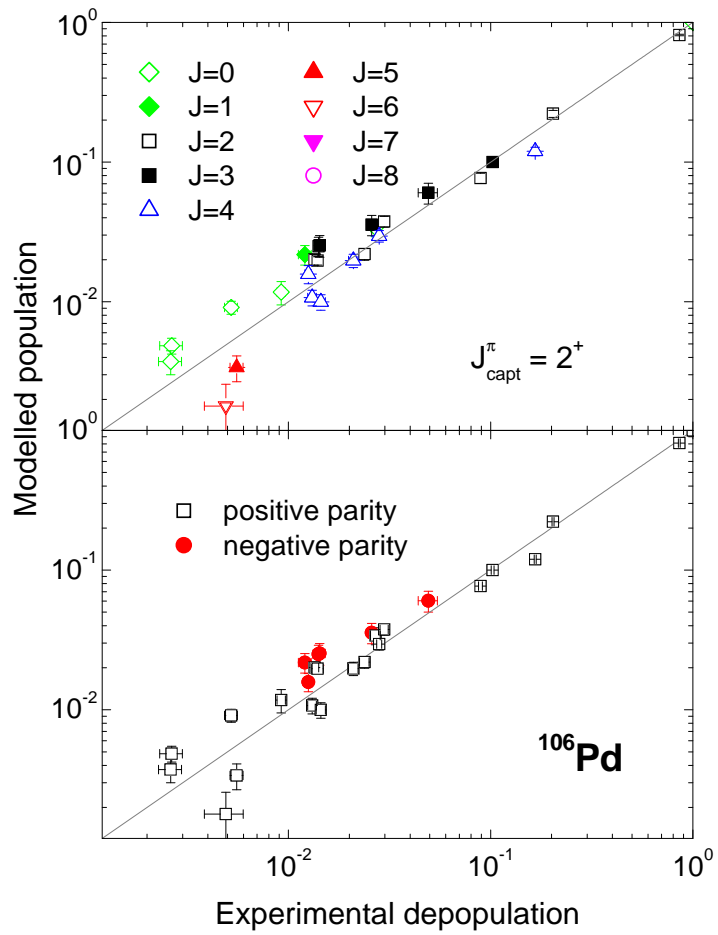

FIGURE 1. Experimental and statistical side feedings for ${ }^{105} \operatorname{Pd}(\mathrm{n}, \gamma)$ assuming the capture state $\mathrm{J}^{\pi}=2^{+}$.

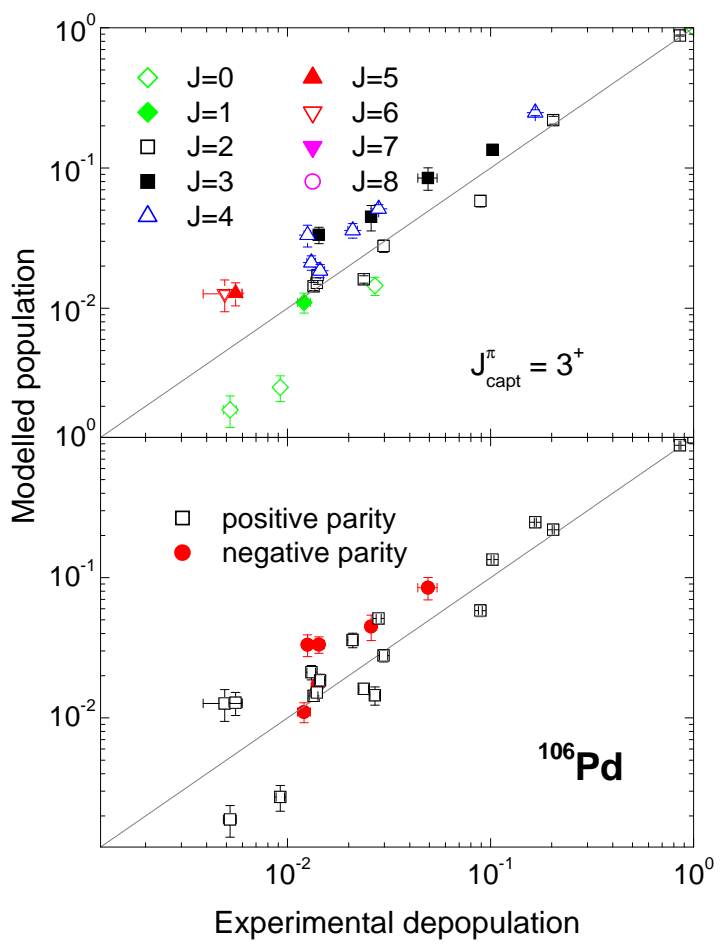

FIGURE 2. Experimental and statistical side feedings for ${ }^{105} \operatorname{Pd}(\mathrm{n}, \gamma)$ assuming the capture state $\mathrm{J}^{\pi}=3^{+}$. 


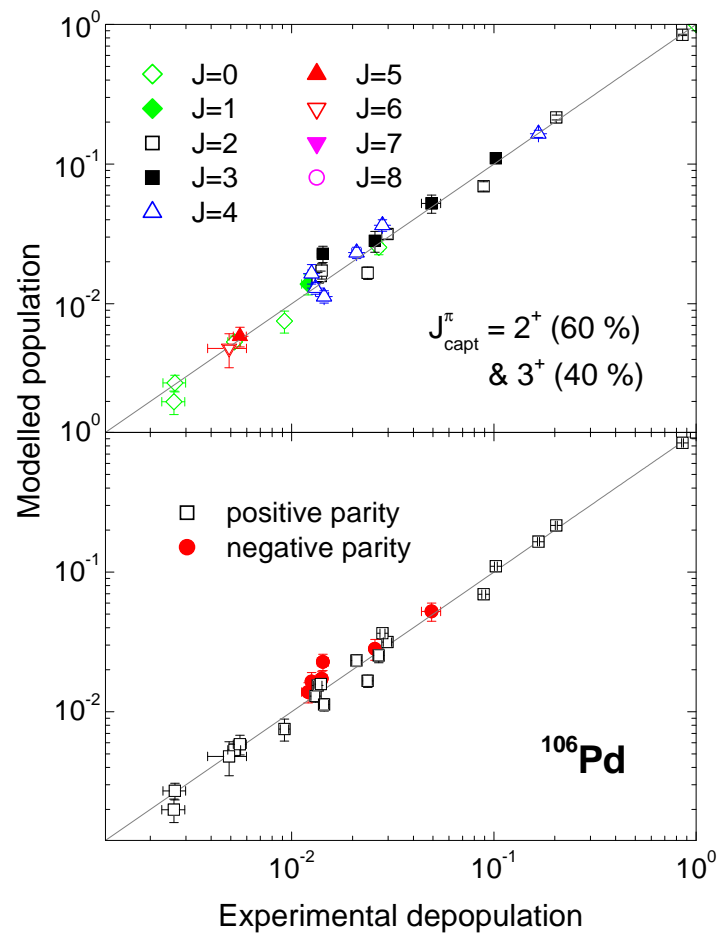

FIGURE 3. Experimental and statistical side feedings for ${ }^{105} \mathrm{Pd}(\mathrm{n}, \gamma)$ assuming the capture state is $2^{+}(60 \%)+3^{+}(40 \%)$

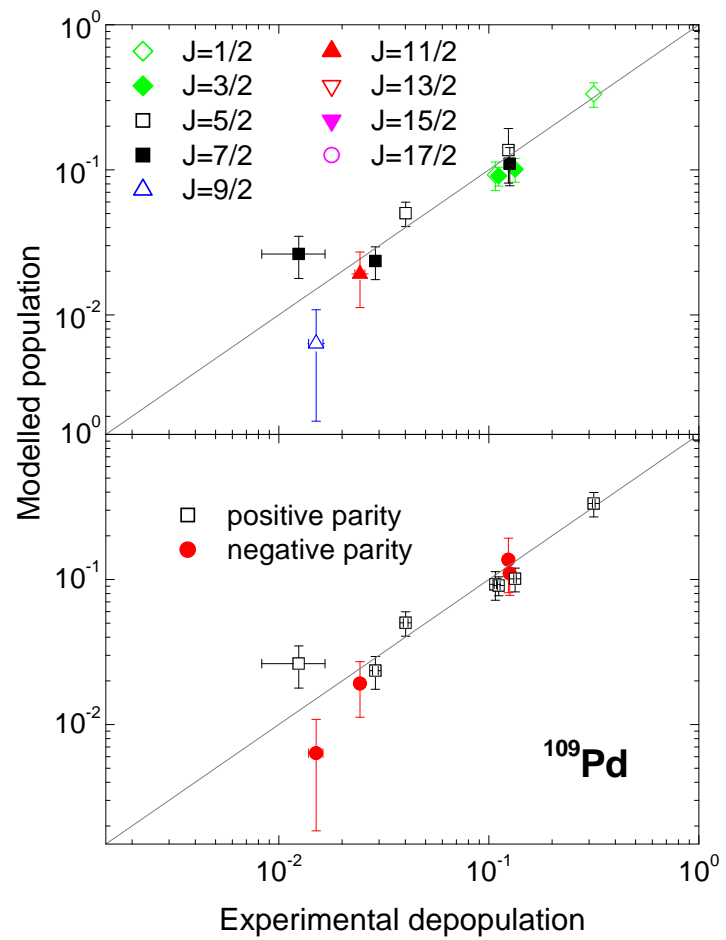

FIGURE 4. Experimental and statistical side feedings for ${ }^{108} \operatorname{Pd}(\mathrm{n}, \gamma)$.
202/03/P136.

\section{REFERENCES}

1. R.B. Firestone, H.D. Choi, R.M. Lindstrom, G.L. Molnár, S.F. Mughabghab, R. Paviotti-Corcuera, Zs. Révay, V. Zerkin. and C.M. Zhou, Database of Prompt Gamma Rays from Slow Neutron Capture for Elemental Analysis, International Atomic Energy Agency TECDOC, in press (2003)

2. F. Becvár, Nucl. Instr. Meth. A417, 434 (1998). 105, 113 (2000).

3. T. Belgya, Zs. Révay, B. Fazekas, I. Héjja, L. Dabolczi, G.L. Molnár, Z. Kis, J. Östör, and Gy. Kaszás, Proc. 9th Intern. Symp. on Capture Gamma-Ray Spectroscopy and Related Topics, Budapest, Hungary, Oct. 8-12, G.L. Molnár, T. Belgya, Zs. Révay (Eds), Springer Verlag, Budapest, 826 (1997).

4. G.L. Molnár, Zs. Révay, and T. Belgya, Nucl. Instrum. Meth. Phys. Res. A489, 140 (2002).

5. B. Fazekas, J. Östör, Z. Kis, G.L. Molnár, and A. Simonits, Proc. 9th Intern. Symp. on Capture GammaRay Spectroscopy and Related Topics, Budapest, Hungary, Oct. 8-12, G.L. Molnár, T. Belgya, Zs. Révay (Eds), Springer Verlag, Budapest, 774 (1997).

6. Evaluated Nuclear Structure Data File, a computer file of evaluated experimental nuclear structure data maintained by the National Nuclear Data Center, Brookhaven National Laboratory.

7. A. Gilbert and A.G.W. Cameron, Can. J. Phys. 43, 1466 (1965).

8. H.A/ Bethe, Rev. Mod. Phys. 9, 69 (1937).

9. W. Dilg, W. Schantl, H. Vonach, and M. Uhl, Nucl. Phys. A217, 269 (1973).

10. P. Axel, Phys. Rev. 126, 671 (1962).

11. J. Kopecky and R.E. Chrien, Nucl. Phys. 468, 285 (1987).

12. S.G. Kadmenskij, V.P. Markushev and V.I. Furman, Sov. J. Nucl. Phys. 37, 165 (1983).

13. S.F. Mughabghab, Thermal Neutron Capture Cross Sections, Resonance Integrals, and g-factors, INDC(NDS)-440 (2003).

14. C.L. Duncan and K.S. Krane, Phys. Rev. C71, 054322 (2005).

15. E.S. Macias, M.E. Phelps, D.G. Sarantites, and R.A. Meyer, Phys. Rev. C14, 639 (1976).

16. W.H. Zoller, E.S. Macias, M.B. Perkal, and W.B. Walters, Nucl. Phys. A130, 293 (1969).

17. M.L. Sehgal, H.S. Hans, and P.S. Gill, Nucl. Phys. 12, 261 (1959). 